\title{
Comparison of Whole and Sectioned Vertebrae for Determining the Age of Young Blue Shark (Prionace glauca)
}

\author{
M. A. MacNeil ${ }^{1}$ \\ Department of Biology, Dalhousie University \\ Halifax, Nova Scotia, Canada B3H 3J5. \\ and \\ S. E. Campana \\ Marine Fish Division, Bedford Institute of Oceanography \\ P.O. Box 1006, Dartmouth, Nova Scotia, Canada B2Y 4A2.
}

\begin{abstract}
Age determinations using whole and sectioned vertebrae from 185 blue shark (Prionace glauca) showed no systematic between-method difference in ages to a minimum age of 8 years. The precision measure, coefficient of variation, was good for both methods ( $\mathrm{CV}<8 \%$ ). While age validation is still required, digitally-enhanced images of whole vertebrae appear to be a rapid and cost effective means of age determination for blue shark below $300 \mathrm{~cm}$ (fork length) or approximately age 8 .
\end{abstract}

Keywords: age-bias, age determination, precision, Prionace glauca vertebrae

\section{Introduction}

The blue shark (Prionace glauca) is among the widest ranging of all shark species, being found in every temperate and tropical ocean (Compagno, 1984). The species is thought to grow quickly, maturing at approximately $185 \mathrm{~cm}$ (fork length: $\mathrm{FL}$ ) or about age 5 (Pratt, 1979). However, estimated growth rates have varied among studies, possibly because of the range of ageing methods which have been applied (Aasen, 1966; Cailliet et al., 1983; Stevens, 1975; Tanaka et al., 1990). Although the accuracy of the age interpretations has been validated in several shark species, there is as yet no validated method of age determination for blue sharks (Natanson and Cailliet, 1990; Brown and Gruber, 1988).

Most length-at-age information for shark species worldwide has come from the interpretation of growth bands visible in vertebral structures (Cailliet, 1987). Past authors have examined whole and/or sectioned vertebrae in their studies (Tanaka et al., 1990; Cailliet et al., 1983; Stevens, 1975). These differences in method may contribute to the apparent differences in growth rate observed among blue shark ageing studies.

The objective of this study was to compare age determinations of blue shark based on whole and sectioned vertebrae using image analysis methods. For simplicity we will use the term ages to denote our age estimates as the ageing of blue shark vertebrae has not yet been validated.

\section{Materials and Methods}

Samples of blue shark vertebrae were obtained from 185 fish caught during sport-fishing tournaments in Nova Scotian waters off the east coast of Canada in August-September 2000. At dockside, sharks were measured (FL, measured over the body curve) to the nearest $\mathrm{cm}$, maturity assessed and vertebral samples were taken from each fish within hours of being caught. Cervical vertebrae ( $n \sim 4)$ adjacent to the most posterior of the gill slits, were removed from each shark upon landing, then frozen for transportation and storage at $-27^{\circ} \mathrm{C}$. In the laboratory, vertebrae were cleaned of adhering soft tissue using a scalpel. Digital

\footnotetext{
${ }^{1}$ Current address: Fisheries Sciences, Warnell School of Forest Resources, University of Georgia, Athens, Georgia, USA 30602.
} 
images of each whole vertebral face were obtained under reflected light using a Kodak Megaplus 1.4 digital camera at a resolution of $1280 \times 1024$ pixels and Optimas 6.1 video capturing software. After whole images had been collected, the vertebrae were sectioned along their midsagittal axis to a thickness of $0.7 \mathrm{~mm}$ with an Isomet slow speed saw and digitally photographed under reflected light. Vertebral images were subsequently enhanced using Adobe Photoshop 5.0 and aged in a blind/independent manner by two readers. Whole vertebrae were aged independently of sectioned vertebrae.

Age-bias plots were generated for analysis of whole/sectioned, inter-ager, and intra-ager results. Age-bias plots provide a clear, simple means of detecting bias and are excellent for illustrating matched pair relationships. Matched pairs are compared by plotting the mean counts of one ager for the samples corresponding to each of the age categories of the other ager. A 1:1 line of equivalence is included to indicate where ager $x=$ ager $y$. The extent of deviation of the $95 \%$ confidence interval bars from the 1:1 equivalence line indicates the extent of ageing bias (see Campana et al., 1995).

Precision estimates for each method of each ager were calculated using the coefficient of variation (CV) as per Chang (1982). Once all vertebral ageing was complete, 30 whole and 30 section images were randomly taken and re-aged by both agers to obtain replicate values for the calculation of $\mathrm{CV}$. The $\mathrm{CV}$ was calculated as:

$$
C V_{j}=100 \times \frac{\sqrt{\sum_{i=1}^{R} \frac{\left(X_{i j}-X_{j}\right)^{2}}{R-1}}}{X_{j}}
$$

where $X_{i j}$ was the $i$ th age determination of the $j$ th fish, $X_{j}$ was the mean age of the $j$ th fish and $R$ was the number of times each fish was aged. This equation was then averaged among all fish to give a mean $\mathrm{CV}$ for both the whole and sectioned methods per ager.

Growth estimates were calculated based on the von Bertalanffy growth model (von Bertalanffy, 1938; Beverton and Holt, 1957):

$$
L_{t}=L_{\infty} *\left[1-\exp \left(-K^{*}\left(t-t_{0}\right)\right)\right]
$$

where $L_{t}$ is length at age $t, L_{\infty}$, is the asymptotic length, $K$ is the growth rate parameter and $t_{0}$ is a theoretical age at which the length is equal to zero. All lengths for comparison with other published von Bertalanffy estimates were first converted to straight total length (TL) using the conversion formula: $\mathrm{TL}=(\mathrm{FL}-$ 1.73872)/0.82995 (Pratt, 1979).

\section{Results}

The lengths of the male blue sharks examined ranged between 147-282 cm, but were bimodally distributed with peaks at 170 and $240 \mathrm{~cm}$ (Fig. 1). Female blue sharks were smaller, ranging between 145$195 \mathrm{~cm}$ and a mode at $170 \mathrm{~cm}$.

Both the whole and sectioned vertebrae (Fig. 2) showed banding patterns of decreasing width from the centre to the edge of each vertebra. The high magnification of the section images allowed for easy resolution of the thinnest bands near the vertebral edge whereas these bands were somewhat compressed in the whole images. Conversely, bands in the whole images were better defined than the diffuse pattern observed in the section images.

The age-bias plot comparing sectioned versus whole ages (Fig. 3) showed no consistent bias between the two ageing methods across the age range of 2-8 years. Therefore, whole and sectioned vertebrae provided comparable age estimates, at least on average. However, the widths of the confidence intervals for ages 2, 7 and 8 were relatively broad, suggesting that the level of agreement for the youngest and oldest ages was considerably less precise than that for the intermediate ages. The precision estimates (CV) were 5.9\% for the section method and $7.7 \%$ for the whole method suggesting they are both highly reproducible.

The von Bertalanffy growth parameters based on whole and section ages (males and females pooled) were similar in magnitude to those reported in other studies (Aasen, 1966; Stevens, 1975; Cailliet et al., 1983; Tanaka et al., 1990; Henderson et al., 2001). Calculated von Bertalanffy growth parameters were $L_{\infty}=300 ; K=0.68$ and $t_{o}=-0.25$ for whole ages and $L_{\infty}=302 ; K=0.58$ and $t_{o}=-0.24$ for section ages. The resulting von Bertalanffy growth curves were similar for whole and section ages (Fig. 4).

\section{Discussion}

The comparison between whole and sectioned vertebrae indicated that the two methods produced comparable ages until at least age 8 , which corresponded to lengths beyond the reported size of sexual maturity (Pratt, 1979). However, inspection of whole vertebrae suggested that inadequate resolution of the narrow 


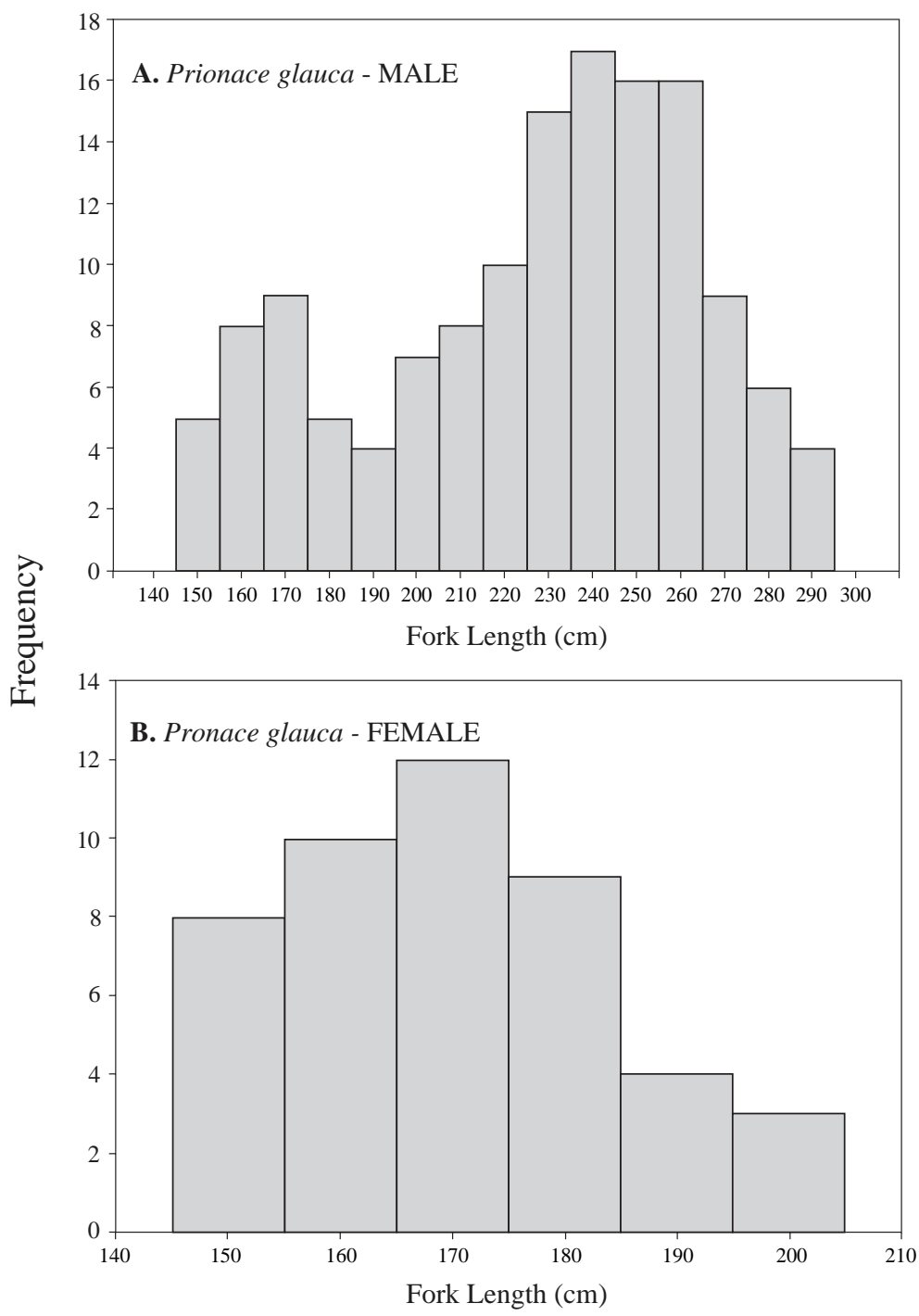

Fig. 1. Length-frequency distribution of sampled (A) male and (B) female blue shark from Nova Scotian waters.

outer bands could be a possible source of ageing bias in older sharks. Blue shark growth declines in older individuals, as is typical for most fishes. Because of the curved surface of the vertebral face, the decreasing width of the most recently-formed growth bands were more difficult to distinguish, resulting in the possible aggregation of adjacent bands during ageing. Age comparisons within and between age readers showed that this had indeed occurred with the less experienced ager in the study, whereby the whole counts of the one ager were shown to be under-aged relative to both the section counts and the counts of the other ager. Presumably this type of error would be less likely with experienced agers. Nevertheless, given these results, we cannot discount the possibility that the whole vertebrae might under-estimate ages in blue sharks older than age 9 .

The length-frequency distributions of both males and females were characterized by modes around 170 $\mathrm{cm}$, corresponding to immature sharks. In the case of the females, this was the only mode present. However the length distribution for males was bimodal, with the larger mode corresponding to mature animals. Thus mature females appear to be absent from Nova Scotian waters during the late summer months.

The ageing precision based on sections (5.9\%) was slightly better than that based on whole vertebrae (7.7\%), although both whole and sectioned vertebrae 

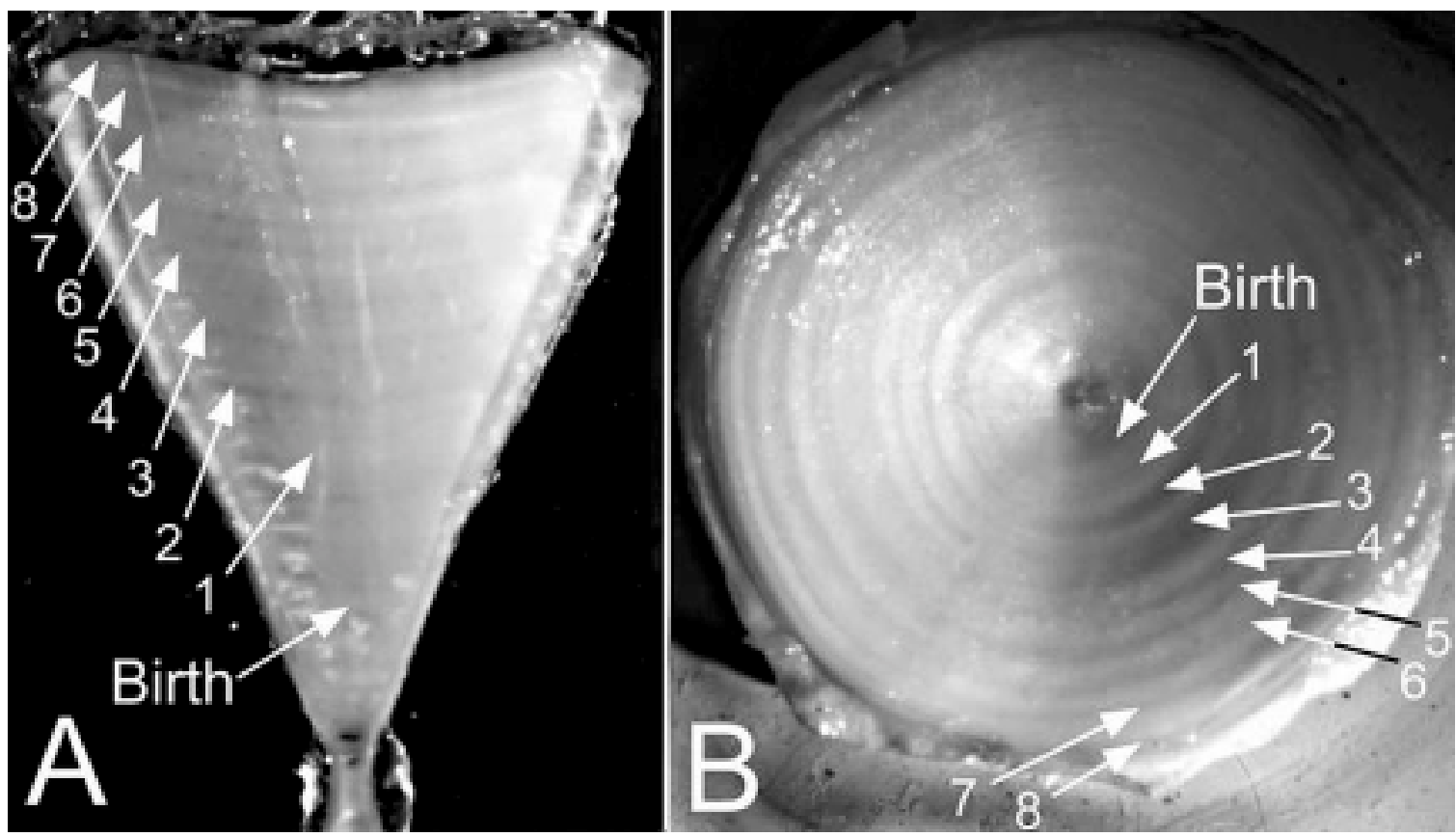

Fig. 2. Images of a single Prionace glauca vertebra under reflected light in both (A) sectioned and (B) whole form. Age determinations are annotated and the birth band noted.

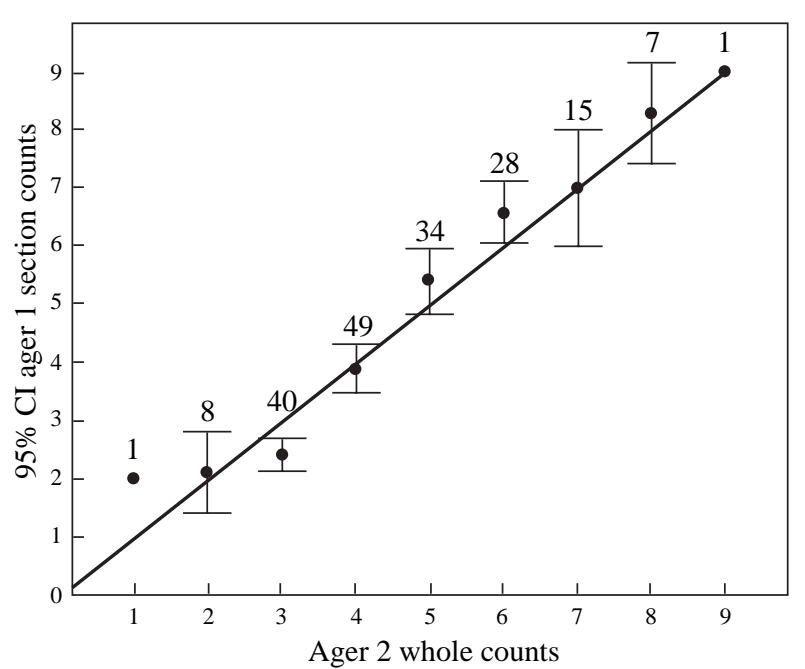

Fig. 3. Age-bias plot comparing ages from whole and sectioned blue sharks vertebrae. The extent of deviation of the $95 \%$ confidence interval bars from the $1: 1$ line indicates the extent of ageing bias.

resulted in more precise ages than the 9-10\% mean $\mathrm{CV}$ value of past vertebra-based studies (Campana, 2001). Therefore both methods were highly reproducible, and when combined with the age-bias results, suggest that they provide analogous ageing rigor.

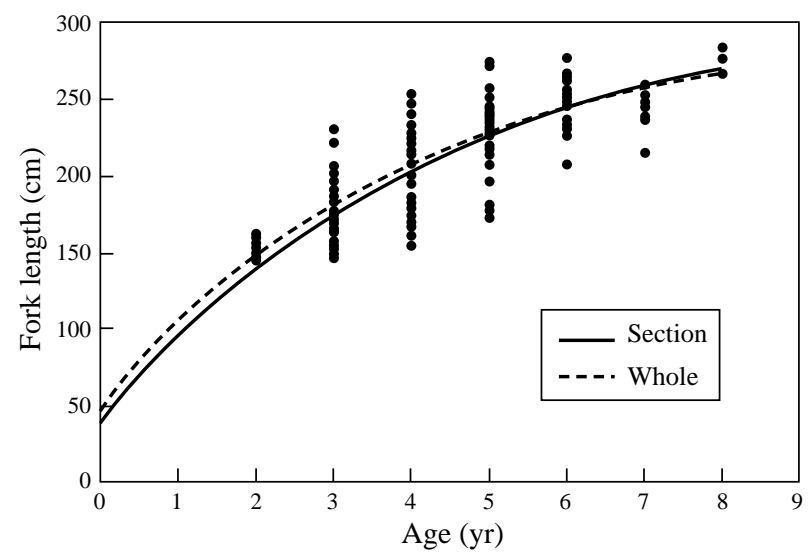

Fig. 4. Von Bertalanffy growth curves for 185 blue sharks (sexes combined) based on whole and sectioned vertebrae. The calculated von Bertalanffy growth equations are: $L_{t}=300(1-\exp [-0.68(t+0.25)])$ for whole ages and $L_{t}=302(1-\exp [-0.58(t+0.24)])$ for section ages.

These results were consistent with those of Tanaka et al. (1990), who found no consistent differences between stained whole vertebrae and vertebral sections, although they did find differences among laboratories. 
In calculating von Bertalanffy growth parameters for our study, we chose to group the male and female data together, based on the absence of mature females. We felt this to be appropriate as studies which have separated males and females have produced inconsistent differences in parameter estimates between sexes, e.g. some show higher $L_{\infty}$ values for males (Cailliet et al., 1983; Tanaka et al., 1990) and others for females (Skomal, MS 1989). The grouped parameters calculated in this study did nonetheless, appear to be similar in range to other studies (Aasen, 1966; Skomal, 1989; Stevens, 1975; Cailliet et al., 1983; Tanaka et al., 1990; Henderson et al., 2001). Plotted curves gave comparable results for both whole and section ages (Fig. 4), an unsurprising result given the close agreement noted by the age bias plots. These curves and estimates are provisional however, as there were few older individuals in our study and no validation has been done. They served principally to ensure our estimates did not differ wildly from previous work.

Tanaka et al. (1990) demonstrated that intra- and inter-reader error, as well as differences among methods, could account for most differences in age interpretation. As outlined above, our intra- and interreader precision was minimal, thereby removing these as substantive sources of variation. Tanaka et al. (1990) also acknowledged inter-oceanic growth differences as a possible source of age estimation differences. Seasonality of temperature and growth in temperate areas are thought to cause the strong banding patterns observed in many temperate species (Stevens, 1975). However, since all previous studies have been carried out in temperate waters (Cailliet et al., 1983; Stevens, 1975; Aasen, 1966; Henderson et al., 2001; Tanaka et al., 1990) it is not known if distinct banding is also present in tropical blue shark. In the absence of age validation studies, our agreement with other studies may be due more to similarities in method than proximity to true ages; i.e. precision does not denote accuracy (Campana, 2001). Until validation work is completed on blue shark age estimates, the accuracy of all reported results will continue to be unknown.

The high resolution digital images produced with the image analysis system provided excellent detail and clarity for ageing purposes and avoided the need for dyes or stains (e.g. Cailliet et al., 1983). Use of whole vertebrae proved to be the easier of the two ageing methods; the resulting images showed clear bands which were acquired in a short period of time using less specialised equipment than those required for the sections. Given the similar ages produced by the two ageing methods, any age validation study which confirms the accuracy of one method must necessarily confirm the accuracy of both methods. Therefore, it is age validation which is now required for age determination of young blue shark.

\section{Acknowledgements}

We thank W. Joyce for assistance with data collection.

\section{References}

AASEN, O. 1966. Blahaien, Prionace glauca (Linnaeus). Fisken Hav., 1: 1-15.

BROWN, C. A. and S. H. GRUBER. 1988. Age assessment of the lemon shark, Negaprion brevirostris, using tetracycline validated vertebral centra. Copeia, 1988(3): 747-753.

BEVERTON, R. J. H. and S. J. HOLT. 1957. On the dynamics of exploited fish populations. Fish. Invest. Minist. Agric. Fish. Food, U.K., 19: 1-533.

CAIlliet, G. M, L. K. MARTIN, J. T. HARVEY, D. KUSHER and B. A. WELDEN. 1983. Preliminary studies on the age and growth of blue Prionace glauca, common thresher, Alopias vulpinus, and shortfin mako, Isurus oxyrinchus, sharks from California waters. In: Proceedings of the international workshop on age determination of oceanic pelagic fishes: tunas, billfishes, and sharks. E. D. Prince and L.M. Pulos (eds.). NOAA Tech. Rep., NMFS, 8: $179-188$.

CAILLIET, G. M. 1987. Estimating age and growth in sharks. In: Sharks: an inquiry into biology, behavior, fisheries, and use. S. Cook (ed.). Oregon State University Extension Service, Portland, Oregon, p.19-27.

CAMPANA, S. E. 2001. Accuracy, precision and quality control in age determination, including a review of the use and abuse of age validation methods. J. Fish. Biol., 59: 197-242.

CAMPANA, S. E., M. C. ANNAND and J. I. MCMILLAN. 1995. Graphical and statistical methods for determining the consistency of age determinations. Trans. Am. Fish. Soc., 124: 131-138.

CHANG, W. Y. B. 1982. A statistical method for evaluating the reproducibility of age determination. Can. J. Fish. Aquat. Sci., 39: 1280-1210.

COMPAGNO, L. V. J. 1984. Sharks of the world. An annotated and illustrated catalogue of shark species known to date. FAO Species Catalogue, Vol. 4, Part 2. Carcharhiniformes. U.N. Dev. Prog., FAO, Rome.

HENDERSON, A. C., K. FLANNERY and J. DUNNE. 2001. Observations on the biology and ecology of the blue shark in the North-east Atlantic. J. Fish. Biol., 58: 1347-1358.

NATANSON, L. J. and G. M. CAILLIET. 1990. Vertebral growth zone deposition in Pacific angel sharks. Copeia, 1990: 1133-1145. 
PRATT, H.L. Jr. 1979. Reproduction in the blue shark, Prionace glauca. Fish. Bull., 77: 445-469.

SKOMAL, G. B. MS 1989. Age and growth of the blue shark, Prionace glauca, in the North Atlantic. M.Sc. Thesis. University of Rhode Island, RI, USA.

STEVENS, J. D. 1975. Vertebral rings as a means of age determination in the blue shark (Prionace glauca L.). $J$. Mar. Biol. Ass. U.K., 55: 657-665.

TANAKA, S., G. M. CAILLIET and K. G. YUDIN. 1990.
Differences in growth of the blue shark, Prionace glauca: Technique or Population? In: Elasmobranchs as Living Resources: Advances in the Biology, Ecology, Systematics, and the Status of the Fisheries. H. L. Pratt Jr., S. H. Gruber and T. Taniuchi (eds.). NOAA Tech. Rep., NMFS, 90: 177187.

VON BERTALANFFY, L. 1938. A quantitative theory of organic growth (inquiries on growth laws. II). Human Biol., 10: 181-213. 